\title{
115. The Gauss Map in Models
}

\author{
By Hiroo Matsuda \\ Department of Mathematics, Kanazawa Medical University \\ (Communicated by Kunihiko KodAIRA, M. J. A., Oct. 12, 1983)
}

1. Introduction. Let $N$ be an $n$-dimensional Riemannian manifold isometrically immersed into a Euclidean $(n+k)$-space $E^{n+k}(k \geqq 1)$ and $C V_{E}(N)$ be the unit normal bundle of $N$ in $E^{n+k}$. Then the Gauss map of $C V_{E}(N)$ into the unit sphere about the origin of $E^{n+k}$ was given by Chern and Lashof [1]. J. L. Weiner [5] gave a generalization of this map as follows: Let $N$ be an isometrically immersed $n$-dimensional Riemannian manifold into a complete $(n+k)$-dimensional Riemannian manifold. Suppose that for a point $p$ of $N, N$ does not intersect the cut locus of $p$. The parallel displacement of $v \in C V_{M}(N)$ (=the unit normal bundle of $N$ in $M$ ) along the shortest geodesic segment joining the foot point of $v$ to $p$ gives a mapping of $C V_{M}(N)$ into the unit sphere in the tangent space of $M$ at $p$. This map is called the Gauss map on $N$ based at $p$. R. Takagi [4] described an $n$-dimensional complete Riemannian $N$ isometrically immersed into a Euclidean $(n+1)$-sphere $S^{n+1}$ when the Gauss map on $N$ based at a point $S^{n+1}$ has constant rank. Furthermore, J. L. Weiner [5] showed similar results when the ambient space is a hyperbolic space of curvature -1 and also reproved Takagi's theorem in a simpler fashion. When the ambient space $M$ is a model with a pole $o$, the cut locus of $o$ is empty. So, for any isometrically immersed Riemannian manifold $N$ into $M$, the Gauss map $G_{M}$ on $N$ based at $o$ can be defined. In this note, we will study the Gauss map $G_{M}$ and show the similar results to those of J. L. Weiner.

2. Preliminaries. Let $(M, o)$ be an $n$-dimensional model with a pole $o(n \geqq 2)$ and $h:=\operatorname{Exp}_{o}: M_{o} \rightarrow M$ be the exponential map from the tangent space $M_{o}$ at $o$ of $M$ onto $M$. Choosing an orthonormal basis $\left\{e_{1}, \cdots, e_{n}\right\}$ on $M_{o}$, let $\left\{y^{1}, \cdots, y^{n}\right\}$ be the normal coordinate system relative to this basis. Let $g$ be the Riemannian metric on $M$. Then $h^{*} g$ is a Riemannian metric on $M_{o}$ and written by

$$
h^{*} g=d r^{2}+f(r)^{2} d \Theta^{2} .
$$

Here $d \Theta^{2}$ denotes the canonical metric on the unit sphere of $M_{o}, r$ is the usual radial function on $M_{o}$ and $f(r)$ is the $C^{\infty}$ function on $[0, \infty)$ satisfying

$$
f(0)=0, f^{\prime}(0)=1, f(r)>0 \quad \text { for } r>0 .
$$

3. Parallel displacements. For a tangent vector 


$$
A=\sum_{i=1}^{n} a^{i}(p) \partial / \partial y^{i}(p) \text { at } p=h\left(\sum_{i=1}^{n} y^{i} e_{i}\right) \in M,
$$

the parallel displacement of $A$ from $p$ to $o$ along the geodesic segment $\zeta$ joining $o$ and $p$ is denoted by $\Gamma(A)$. Then, by the use of Jacobi fields, we have

Lemma 1.

$$
\Gamma(A)=\frac{f(r)}{r} \sum_{i=1}^{n} a^{i}(p) e_{i}+\left(1-\frac{f(r)}{r}\right) g(A, \dot{\zeta}(r)) \sum_{i=1}^{n} \frac{y^{i}}{r} e_{i}
$$

where $r=\left(\sum_{i=1}^{n}\left(y^{i}\right)^{2}\right)^{1 / 2}=$ the distance between $o$ and $p$.

Remark 1. We note that $g(A, \dot{\zeta}(r))=\sum_{i=1}^{n} a^{i}(p)\left(y^{i} / r\right)$ by the Gauss Lemma.

Now, we define the new coordinate system on $M$ by

$$
x^{i}(p):=\frac{y^{i}(p)}{r} \exp \left(\int_{1}^{r} \frac{d s}{f(s)}\right) \quad(i=1, \cdots, n)
$$

for $p \in M$, where $r=\left(\sum_{i=1}^{n}\left(y^{i}(p)\right)^{2}\right)^{1 / 2}=$ the distance between $o$ and $p$. It is shown as follows that $\left\{x^{1}, \ldots, x^{n}\right\}$ is a coordinate system of $M$.

Lemma 2. The map $\Psi: M \rightarrow R^{n}$ defined by $\Psi:=u \circ h^{-1}$ is one to one and $C^{\infty} \operatorname{map}$, where $u$ is the $\operatorname{map} M_{o} \rightarrow R^{n}$ defined by

and

$$
u\left(\sum_{j=1}^{n} y^{j} e_{j}\right)=\left(x^{1}, \cdots, x^{n}\right)
$$

$$
x^{i}:=\frac{y^{i}}{r} \exp \left(\int_{1}^{r} \frac{d s}{f(s)}\right), \quad r=\left(\sum_{i=1}^{n}\left(y^{i}\right)^{2}\right)^{1 / 2} .
$$

Proof. Since $h^{-1}: M \rightarrow M_{0}$ is diffeomorphism, it is sufficient to show that $u$ is one to one and $C^{\infty}$ map. It is clear that $u$ is one to one on $M_{o}$ and $C^{\infty}$ in $M_{o}-\{0\}$. Let

$$
F\left(\sum_{j=1}^{n} y^{j} e_{j}\right):=(1 / r) \exp \left(\int_{1}^{r} \frac{d s}{f(s)}\right) \text {. }
$$

Since

$$
\partial F / \partial y^{i}=F \frac{y^{i}}{r^{2}}\left(\frac{r}{f(r)}-1\right)
$$

and $\lim _{r \rightarrow 0} F$ is uniquely determined as to be a positive constant, we must show that each

$$
\eta^{i}:=\frac{y^{i}}{r^{2}}\left(\frac{r}{f(r)}-1\right)
$$

has a smooth extension across the origin. It is known ([2], [3]) that $f(r)=r+r^{3} l(r)$ and $l(r)$ has a smooth extension across the origin. Thus

$$
\eta^{i}=\frac{y^{i}}{r^{2}}\left(\frac{r}{r+r^{3} l(r)}-1\right)=\frac{-y^{i} l(r)}{1+r^{2} l(r)}
$$

has a smooth extension across the origin.

Now we have the parallel translation in terms of the new coordinate system $\left\{x^{i}\right\}$ by Lemma 1 and Remark 1 .

Lemma 3. For a tangent vector $A=\sum_{i=1}^{n} b^{i}(p)\left(\partial / \partial x^{i}\right)(p)$ at $p \in M$, we have 
No. 8]

$$
\Gamma(A)=\frac{F(0) f(r)}{\exp \left(\int_{1}^{r} \frac{d s}{f(s)}\right)} \sum_{i=1}^{n} b^{i}(p) \frac{\partial}{\partial x^{i}}(o)
$$

where $r=$ the distance between $o$ and $p$, and

$$
F(0)=\lim _{r \rightarrow 0}(1 / r) \exp \left(\int_{1}^{r} \frac{d s}{f(s)}\right)=\lim _{r \rightarrow 0}(1 / f(r)) \exp \left(\int_{1}^{r} \frac{d s}{f(s)}\right) .
$$

Now let $\rho=\exp \left(\int_{1}^{r} \frac{d s}{f(s)}\right)$. Then $\rho=\left(\sum_{i=1}^{n}\left(x^{i}\right)^{2}\right)^{1 / 2}$ and so by Lemma 3, we have

Proposition. Let $(M, o)$ be an n-dimensional model with a pole o. Then

(1) If $\exp \left(\int_{1}^{\infty} \frac{d s}{f(s)}\right)=\rho_{0}<\infty, M$ is isometric to

$$
D^{n}=\left\{\left(x^{1}, \cdots, x^{n}\right) \mid \sum_{i=1}^{n}\left(x^{i}\right)^{2}=\rho_{0}^{2}\right\}
$$

with the Riemannian metric $\gamma(\rho)^{2} g_{0}$ where $g_{0}$ is the restriction of the canonical Euclidean metric on $R^{n}$ to $D^{n}$.

(2) If $\exp \left(\int_{1}^{\infty} \frac{d s}{f(s)}\right)=\infty, M$ is isometric to $R^{n}$ with the Riemannian metric $\gamma(\rho)^{2} g_{0}$.

4. The Gauss map. Let $N$ be an $n$-dimensional Riemannian manifold isometrically immersed in an $(n+k)$-dimensional model $(M, 0)$ with a pole $o(k \geqq 1)$. Then, by Proposition in $\S 3, N$ may be immersed (not isometrically in general) in the Euclidean space $E^{n+k}$ of dimension $n+k$. Let $G_{E}$ be the usual Gauss map in $E^{n+k}$. By Lemma 3, we have

Lemma 4. Let $N$ be an n-dimensional Riemannian manifold isometrically immersed in an $(n+k)$-dimensional model $(M, o)$. Then the following diagram is commutative

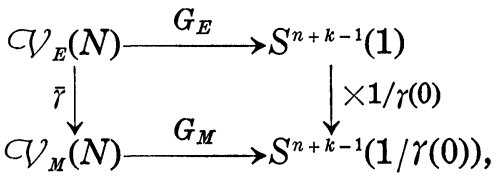

where $\bar{\gamma}(v):=(1 / \gamma(\rho)) v$ for a unit normal vector $v$ at $p=\left(x^{1}, \cdots, x^{n}\right), \rho$ and $\gamma$ are the same as in Proposition, and $S^{n+k-1}(\alpha)$ is the sphere about the origin in $E^{n+k}$ of radius $\alpha$.

Corollary. Since $\bar{\gamma}: C V_{E}(N) \rightarrow C V_{M}(N)$ is a diffeomorphism, the rank of $G_{E}$ at $v$ equals the rank of $G_{M}$ at $\bar{\gamma}(v)$ for all $v \in C V_{E}(N)$.

If $N$ is orientable and $k=1$, we can identify $N$ with a component of $C V_{M}(N)$ and also the corresponding component of $C V_{E}(N)$. Then $G_{M}: N \rightarrow$ the unit $n$-sphere about the origin in $M_{o}$ is the Gauss map based at $o$ and $G_{E}: N \rightarrow S^{n}(1)$ is the usual Gauss map in $E^{n+1}$.

Theorem. Let $N$ be an $n$-dimensional complete orientable Riemannian manifold isometrically immersed in an $(n+1)$-dimensional 
model $(M, o)$. Suppose that $G_{M}$ has constant rank $n-m$ on $N(0 \leqq m \leqq n)$.

(1) If $m=0$ and $N$ is compact, then $N$ is diffeomorphic to the $n$ sphere.

(2) If $1 \leqq m \leqq n-1$, then $N$ is foliated by $m$-dimensional totally umbilic submanifolds.

(3) If $m=n$, then $N$ is a totally umbilic hypersurface.

Proof. (1) is clear.

Since the rank of $G_{M}$ equals the rank of $G_{E}$ by corollary, $N$ is foliated by $m$-dimensional planes $L^{m}$ in $E^{n+1}$ intersected with $M$ by Lemma 2 of [1]. For each $L^{m}, L^{m} \cap M$ with the induced metric from $M$ is a totally umbilic submanifold. Thus (2) and (3) are verified.

Remark 2. If $M$ is the hyperbolic space, each totally umbilic submanifold in Theorem is a hyperbolic space of a certain constant curvature (see [5]).

Acknowledgement. The author wishes to thank Prof. H. Kitahara, S. Yorozu and S. Nagamine for their useful advices.

\section{References}

[1] S. S. Chern and R. K. Lashof: On the total curvature of immersed manifolds. Amer. J. Math., 79, 306-313 (1957).

[2] R. E. Greene and H. Wu: Function Theory on Manifolds Which Possesses a Pole. Lect. Notes in Math., vol. 699, Springer-Verlag (1979).

[ 3 ] J. L. Kazdan and F. W. Warner: Curvature functions for open 2-manifolds. Ann. of Math., 99, 203-219 (1974).

[4] R. Takagi: Gauss map in a sphere. Kōdai Math. Sem. Rep., 22, 82-88 (1970).

[5] J. L. Weiner: The Gauss map in spaces of constant curvature. Proc. Amer. Math. Soc., 38, 157-161 (1973). 